\title{
西双版纳尚勇自然保护区哺乳动物物种多样性
}

\author{
白德凤 ${ }^{1}$ 陈 颖 ${ }^{1}$ 李俊松 $^{2}$ 陶 庆 $^{3}$ 王利繁 ${ }^{2}$ 飘 优 ${ }^{2}$ 时 坤 $1^{*}$ \\ 1 (北京林业大学自然保护区学院野生动物研究所, 北京 100083) \\ 2 (西双版纳国家级自然保护区尚勇管理所, 云南预腊 666300) \\ 3 (西双版纳国家级自然保护区管理局，云南景洪 666100)
}

\section{Mammal diversity in Shangyong Nature Reserve, Xishuangbanna, Yun- nan Province}

Defeng Bai ${ }^{1}$, Ying Chen ${ }^{1}$, Junsong $\mathrm{Li}^{2}$, Qing Tao ${ }^{3}$, Lifan Wang ${ }^{2}$, You Piao ${ }^{2}$, Kun Shi ${ }^{1 *}$

1 Wildlife Institute, School of Nature Conservation, Beijing Forestry University, Beijing 100083

2 Administration Office of Xishuangbanna Shangyong Nature Reserve, Mengla, Yunnan 666300

3 Administration Bureau of Xishuangbanna National Nature Reserve, Jinghong, Yunnan 666100

尚勇自然保护区位于云南省西双版纳预腊县 南部, 地理位置2113'30"-2124'48" N, 10122'48"1013'3" E, 为1980年西双版纳自然保护区调整 后新增保护区域。总面积 $31,184 \mathrm{ha}$, 其中核心区面 积 18,199 ha, 缓冲区面积 6,736 ha, 实验区面积 6,249 ha (杨宇明和唐芳林, 2008)。

过去几十年的人为干扰使得西双版纳热带雨 林破碎化程度加剧, 哺乳动物多样性呈现逐渐降低 的趋势(Pu \& Zhang, 2001; Li et al, 2009)。近年来在 尚勇自然保护区开展的野生哺乳动物研究主要集 中在亚洲象(Elephas maximus)(冯利民和张立, 2005; Chen et al, 2006; 林柳等, 2011; 何长欢等, 2015)、印 支虎(Panthera tigris corbetti) (胡建生等, 1999; 冯 利民等, 2013)、印度野牛(Bos gaurus)(张忠员等, 2016)、威氏小鼠鹿(Tragulus williamsoni) (杨鸿培等, 2017)等具体物种上, 对该地的哺乳动物多样性尚 缺乏较为全面的认识。为此, 我们采用红外相机技 术对该地的哺乳动物进行了为期约 6 个月的调查。

\section{1 研究方法}

2014年1-6月, 在尚勇自然保护区布设了 49台 红外相机(Ltl6210) (图1)。布设时, 相邻2个位点的 直线距离尽可能大于 $1 \mathrm{~km}$ 。红外相机多安放在兽
道、发现兽类痕迹处或者有经验的向导所指示的地 方，离地面高度约40-50 cm。设置为拍照模式，照 片大小为 $8 \mathrm{Mb}$, 触发时三连拍，无拍摄间隔，低灵 敏度，时间、日期与放置相机的时间和日期同步。 红外相机自安放之日起24 h工作, 用碱性电池保证 电量的供应。由于自然损毁和人为干扰(偷盗), 有效 回收红外相机 40 台, 其中 7 台由于雨林湿度较大电 量耗尽未能全时工作。

记录每张哺乳动物照片的拍摄时间及物种信 息，物种的鉴定和分类参考Smith等(2009)和蒋志刚 等(2015)。分布型参考张荣祖(1999)。将30 min内连 续拍摄到的相同物种的照片定义为 1 个独立事件 (independent event)(李晟等, 2016; 汤小明等, 2016)。 以相机工作 $24 \mathrm{~h}$ 为 1 个有效相机日 (effective trap day), 并计算哺乳动物的相对丰富度指数(relative abundance index, $R A I$ ) (Liu et al, 2013), 公式如下:

$$
R A I=A i / N \times 100
$$

其中 $A i$ 代表一个物种被所有相机拍摄到的独立事件 数量, $N$ 表示在研究期间红外相机拍摄到的所有物 种的独立事件总和。

\section{2 结果与分析}

40 台红外相机累计工作 4,671个有效相机日, 


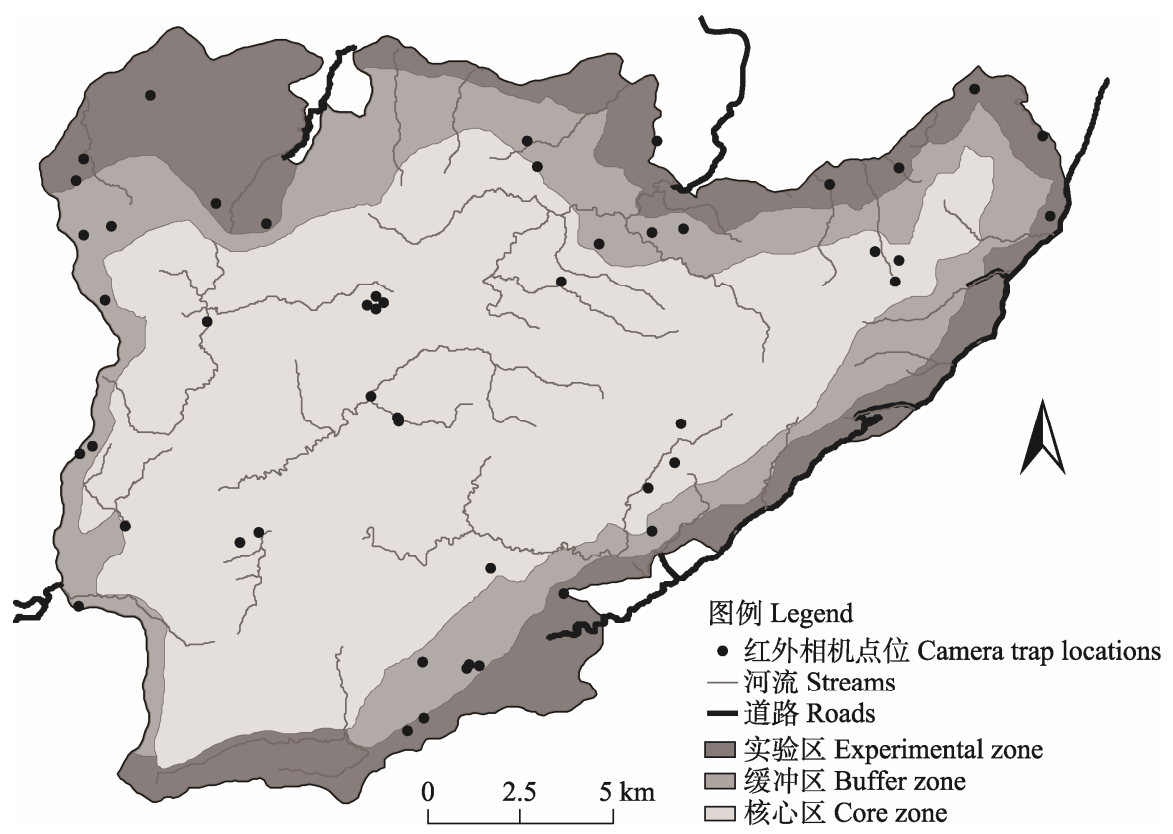

图1 尚勇保护区红外相机布设位点图

Fig. 1 The map of camera locations in Shangyong Nature Reserve

得到18,540张有效照片, 其中野生哺乳动物照片 17,308张(93.35\%), 鸟类照片683张(3.68\%), 爬行类 照片7张(0.038\%), 其他542张(2.92\%)。

经鉴定，哺乳动物有 22 种，隶属于 5 目 15 科 21 属。因啮齿类照片模糊, 无法鉴定到种的均归入啮 齿类未定种。食肉目种数最多(11种), 其次是偶蹄目 (5种)和啮齿目 (3种), 其余拍到灵长目 2 种和长鼻目 1种。北豚尾猴(Macaca leonina)、马来熊(Helarctos malayanus)、熊狸(Arctictis binturong)、亚洲象和威 氏小鼠鹿为国家I级保护动物, 猕猴 (Macaca mulatta)、金猫 (Catopuma temminckii)、楼 (Cuon alpinus)、大灵猫(Viverra zibetha)和马来水鹿(Cervus equinus)为国家II级保护动物。在动物地理区划上, 尚勇自然保护区位于东洋界华南区滇南山地亚区。 从分布型来看, 哺乳动物以东洋型为主(16种), 占 72.73\%; 古北型2种; 喜马拉雅-横断山区型1种; 南 中国型1种; 中亚型1种; 季风型1种。

在拍摄到的哺乳动物中, 偶蹄目照片数量最多, 占照片总数量的62.19\% $(10,764 / 17,308)$; 其次是啮 齿目 $(21.05 \% ; 3,643 / 17,308)$ 、食肉目 $(13.02 \%$; $2,254 / 17,308)$ 、长鼻目 $(2.22 \% ; 384 / 17,308)$ 和灵长目 $(1.52 \% ; 263 / 17,308)$ 。马来水鹿 $(R A I=25.48)$ 、赤鹿 (Muntiacus vaginalis, $R A I=24.79$ )、野猪(Sus scrofa, $R A I=6.93)$ 、威氏小鼠鹿 $(R A I=4.57) 、$ 食蟹獴
(Herpestes urva, $R A I=3.88)$ 、鼠獾 $(R A I=3.74)$ 和亚 洲象 $(R A I=2.22)$ 物种相对丰富度较高。马来水鹿、 赤鹿、野猪和威氏小鼠奚鹿独立事件数最多, 占总的 拍摄数量的 $61.77 \%$ (446/772), 其中马来水鹿和赤 麇的独立事件所占的比例分别高达25\% (184/772) 和24\% (179/772)。调查区域马来水鹿和赤鹿的相对 丰富度之和 $(R A I=50.28)$ 超过其他所有拍摄到哺乳 动物物种的相对丰富度之和。此次调查拍摄到的能 够影响马来水鹿和赤鹿种群的捕食者种类少(仅牧才、 马来熊和金猫), 且相对丰富度较低(类、马来熊和金 猫的 $R A I$ 值依次为 $0.69 、 0.55$ 和 0.14 )。

物种累积曲线显示累积有效相机日在2,218日 以下时, 随着有效相机日的增加, 物种数量增长很 快, 到2,218 个有效相机日时, 红外相机拍摄到 90.9\% (20/22)的哺乳动物物种, 此时曲线趋于平缓, 此结果与张明霞等(2014)在西双版纳的研究结果相 近。累积到3,645 个有效相机日时首次捕捉到豹猫 (Prionailurus bengalensis), 在4,091个有效相机日时 首次捕捉到大灵猫(图2)。此次调查捕捉到的豹猫相 对丰富度较低, 可能反映了豹猫在尚勇自然保护区 的相对数量较少, 也可能是相机布设位点设置位置 较高及红外相机位点不足所致。

西双版纳国家级自然保护区现记录到哺乳动 物10目34科130种(西双版纳自然保护区综合考察团, 


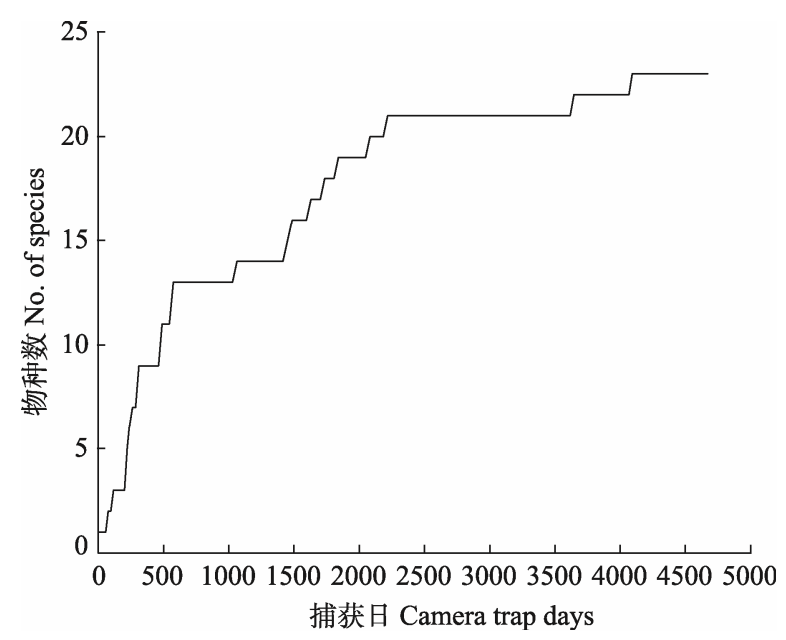

图2 哺乳动物物种累积曲线

Fig. 2 The curve of cumulative number of mammal species

1987; 杨宇明和唐芳林, 2008), 此次仅拍摄到哺乳 动物 5 目 15 科 22 种, 一方面是由于红外相机拍摄到 的啮齿类跑动太快, 根据照片难以鉴定到种, 另一 方面也是因为红外相机位点较少覆盖不全面。本次 调查时隔30年再次用红外相机记录到豹在西双版 纳地区活动, 但其生存现状尚需要进一步的调查研 究。冯利民等(2013)曾在尚勇自然保护区确认存在3 只成年印支虎个体(2雌1雄)。2016年6月, 西双版纳 保护区工作人员在西双版纳东部中国老挝边境地 区拍摄到金钱豹(Panthera pardus)(http://www.forestry.gov.cn/main/443/content-884802.html)。2016年, 宋大昭和王志胜(2016)曾在西双版纳易武州级自然 保护区的中老边境地带拍摄到了云豹 (Neofelis nebulosi)。本次调查并未记录到印支虎、金钱豹和 云豹, 尚勇自然保护区是否存在印支虎、金钱豹和 云豹还需要更多的调查进行确证。未来需要开展长 期且更加全面的调查以了解在尚勇自然保护区是 否存在因缺乏捕食者而导致有蹄类物种数量剧增 的可能。

致谢: 感谢西双版纳国家级自然保护区管理局和尚 勇管理所领导的支持, 感谢尚勇自然保护区的工作 人员赵金清、桑捌、当飘、岩丙、车志勇、周开华、 董志明、李忠云、波糯叫等人在野外工作中提供的 大力支持和帮助, 感谢北京林业大学自然保护区学 院杨子诚对数据整理和录入的帮助以及陈鹏举和 潘国梁对论文修改的建议。

\section{参考文献}

Chen J, Deng XB, Zhang L, Bai ZL (2006) Diet composition and foraging ecology of Asian elephants in Shangyong, Xishuangbanna, China. Acta Ecologica Sinica, 26, 309-316.

Comprehensive Investigation Group of Xishuangbanna Nature Reserve (1987) Comprehensive Survey Report on Xishuangbanna Nature Reserve. Yunnan Science and Technology Press, Kunming. (in Chinese) [西双版纳自然保护 区综合考察团 (1987) 西双版纳自然保护区综合考察报 告集. 云南科技出版社, 昆明.]

Feng LM, Zhang L (2005) Habitat selection by Asian elephant (Elephas maximus) in Xishuangbanna, Yunnan, China. Acta Theriologica Sinica, 25, 229-236. (in Chinese with English abstract) [冯利民, 张立 (2005) 云南西双版纳尚勇保护 区亚洲象对栖息地的选择. 兽类学报, 25, 229-236.]

Feng LM, Wang LF, Wang B, Smith JLD, Zhang L (2013) Population status of the Indochinese tiger (Panthera tigris cobetti) and density of the three primary ungulate prey species in Shangyong Nature Reserve, Xishuangbanna, China. Acta Theriologica Sinica, 33, 308-318. (in Chinese with English abstract) [冯利民, 王利繁, 王斌, James LD Smith, 张立 (2013) 西双版纳尚勇自然保护区野生印支虎及其 三种主要有蹄类猎物种群现状调查. 兽类学报, 33, 308-318.]

He CH, Zhou Y, Wang LF, Zhang L (2015) Estimating population size and genetic diversity of Asian elephant in the Shangyong Nature Reserve. Biodiversity Science, 23, 202-209. (in Chinese with English abstract) [何长欢, 周玉, 王利繁, 张立 (2015) 尚勇保护区亚洲象种群数量评估 和遗传多样性分析. 生物多样性, 23, 202-209.]

Hu JS, Jiang WG, Wu JL, Li ZQ, Li CH (1999) Status of Indo-Chinese tigers in Xishuangbanna, China. Chinese Wildlife, (2), 8. (in Chinese with English abstract) [胡建生, 江 望高, 吴京亮, 李宗强, 李春红 (1999) 西双版纳印支虎 的现状. 野生动物, (2), 8.]

Jiang ZG, Ma Y, Wu Y, Wang YX, Feng ZJ, Zhou KY, Liu SY, Luo ZH, Li CW (2015) China's mammalian diversity. Biodiversity Science, 23, 351-364. (in Chinese with English abstract) [蒋志刚, 马勇, 吴毅, 王应祥, 冯祚建, 周开亚, 刘少英, 罗振华, 李春旺 (2015) 中国哺乳动物多样性. 生物多样性, 23, 351-364.]

Li HM, Ma YX, Liu WJ, Liu WJ (2009) Clearance and fragmentation of tropical rain forest in Xishuangbanna, SW, China. Biodiversity and Conservation, 18, 3421-3440.

Li S, Wang DJ, Bu HL, Liu XG, Jin T (2016) Camera-trapping survey on the mammal diversity of the Laohegou Nature Reserve, Sichuan Province. Acta Theriologica Sinica, 36, 282-291. (in Chinese with English abstract) [李晟, 王大军, 卜红亮, 刘小庚, 靳剘 (2016) 四川省老河沟自然保护区 兽类多样性红外相机调查. 兽类学报, 36, 282-291.]

Lin L, Zhang LT, Luo AD, Wang LF, Zhang L (2011) Population dynamics, structure and seasonal distribution pattern of 
Asian elephant (Elephas maximus) in Shangyong Protected Area, Yunnan, China. Acta Theriologica Sinica, 31, 226-234. (in Chinese with English abstract) [林柳, 张龙田, 罗爱东, 王利繁, 张立 (2011) 尚勇保护区亚洲象种群数 量动态、种群结构及季节分布格局. 兽类学报, 31 , 226-234.]

Liu XH, Wu PF, Melissa S, Cai Q, He XB, Zhu Y, Shao XM (2013) Monitoring wildlife abundance and diversity with infra-red camera traps in Guanyinshan Nature Reserve of Shaanxi Province, China. Ecological Indicators, 33, 121-128.

Pu YS, Zhang ZY (2001) A strategic study on biodiversity conservation in Xishuangbanna. Journal of Forestry Research, 12, 25-30.

Smith A, Xie Y, Hoffmann RS (2009) A Guide to the Mammals of China. Hunan Education Publishing House, Changsha. (in Chinese) [Smith A, 解炎, Hoffmann RS (2009) 中 国兽类野外手册. 湖南教育出版社, 长沙.]

Song DZ, Wang ZS (2016) Clouded leopard in Xishuangbanna. Forest \& Humankind, (11), 142-145. (in Chinese) [宋大昭, 王志胜 (2016) 云豹重现. 森林与人类, (11), 142-145.]

Tang XM, Zhang DH, Ma ZH, Wu TL, Zhang YS, Bao WD (2016) Camera trapping survey on ground-dwelling birds and mammals of spring and winter in Beijing Wulingshan Nature Reserve. Chinese Journal of Zoology, 51, 751-760. (in Chinese with English abstract) [汤小明, 张德怀, 马志 红, 吴同路, 张源笙, 鲍伟东 (2016) 北京雾灵山自然保 护区冬春季地面活动鸟兽红外相机初步调查. 动物学杂 志, 51, 751-760.]
Yang HP, Song ZY, Yang ZC, Yang ZB, Li JS (2017) Study on population distribution of Tragulus jacanicus in Xishuangbanna. Forest Inventory and Planning, 42(4), 23-27, 36. (in Chinese with English abstract) [杨鸿培, 宋志勇, 杨子城, 杨正斌, 李俊松 (2017) 西双版纳鼠奚鹿种群分布研究. 林 业调查规划, 42(4), 23-27, 36.]

Yang YM, Tang FL (2008) Study on the Master Planning of Xishuangbanna National Nature Reserve. Science Press, Beijing. (in Chinese) [杨宇明，唐芳林 (2008) 西双版纳国 家级自然保护区总体规划研究. 科学出版社, 北京.]

Zhang MX, Cao L, Quan RC, Xiao ZS, Yang XF, Zhang WF, Wang XZ, Deng XB (2014) Camera trap survey of animals in Xishuangbanna Forest Dynamics Plot, Yunnan. Biodiversity Science, 22, 830-832. (in Chinese) [张明霞, 曹林, 权 锐昌, 肖治术, 杨小飞, 张文富, 王学志, 邓晓保 (2014) 利用红外相机监测西双版纳森林动态样地的野生动物多 样性. 生物多样性, 22, 830-832.]

Zhang RZ (1999) Zoogeography of China. Science Press, Beijing. (in Chinese) [张荣祖 (1999) 中国动物地理. 科学出 版社, 北京.]

Zhang ZY, Yang HP, Luo AD (2016) Population, distribution and conservation status of Guar (Bos gaurus) in Xishuangbanna Prefecture. Forestry Inventory and Planning, 41(2), 115-119. (in Chinese with English abstract) [张忠员, 杨鸿 培, 罗爱东 (2016) 西双版纳印度野牛种群数量、分布和 保护现状. 林业调查规划, 41(2), 115-119.]

(责任编委: 蒋学龙 责任编辑: 问文杰)

\section{附录 Supplementary Material}

附录1 尚勇自然保护区红外相机拍摄的哺乳动物名录(2014年6月)

Appendix 1 The list of mammals captured by camera traps in Xishuangbanna Shangyong Nature Reserve, Yunnan (June, 2014). http://www.biodiversity-science.net/fileup/PDF/2017223-1.pdf

附录2 尚勇自然保护区红外相机拍摄到的重要哺乳动物照片

Appendix 2 Important mammals recorded by camera traps in Shangyong Nature Reserve http://www.biodiversity-science.net/fileup/PDF/2017223-2.pdf 
白德风，陈颖，李俊松，陶庆，王利繁，飘优，时坤. 西双版纳尚勇自然保护区哺乳动物物种多样性. 生物多样性, 2018, 26 (1): 75-78. http://www.biodiversity-science.net/CN/10.17520/biods.2017223

附录1 尚勇自然保护区红外相机拍摄的哺乳动物名录(2014年6月)

Appendix 1 The list of mammals captured by camera traps in Xishuangbanna Shangyong Nature Reserve, Yunnan (June, 2014).

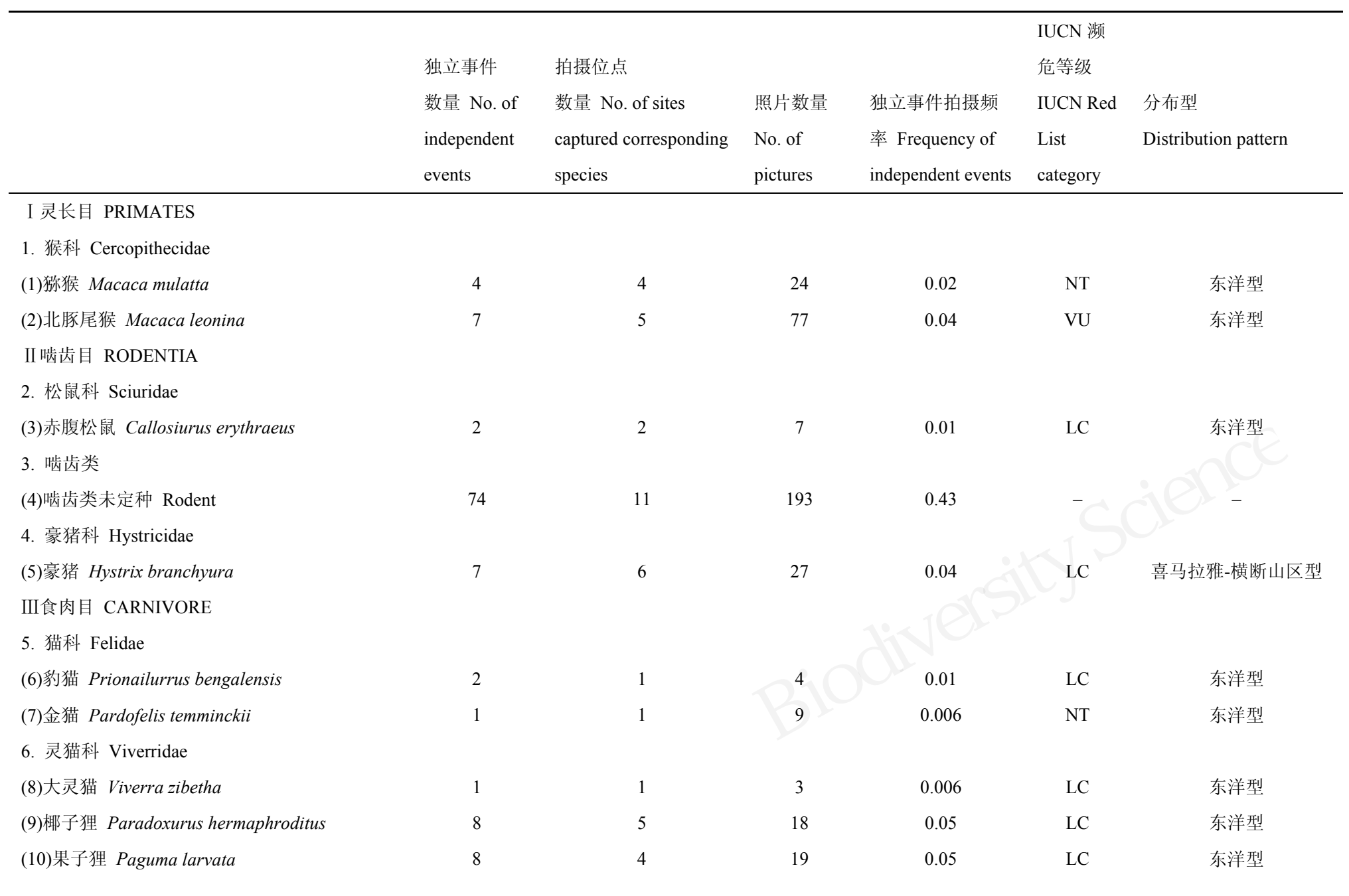


白德风，陈颖，李俊松，陶庆，王利繁，飘优，时坤. 西双版纳尚勇自然保护区哺乳动物物种多样性. 生物多样性, 2018, 26 (1): 75-78. http://www.biodiversity-science.net/CN/10.17520/biods.2017223

\begin{tabular}{|c|c|c|c|c|c|c|}
\hline (11)熊狸 Arctictis binturong & 3 & 3 & 14 & 0.02 & LC & 东洋型 \\
\hline \multicolumn{7}{|l|}{ 7. 㮠科 Herpestidae } \\
\hline (12)食蟹獾 Herpestes urva & 28 & 10 & 96 & 0.16 & 无 & 东洋型 \\
\hline \multicolumn{7}{|l|}{ 8. 犬科 Canidae } \\
\hline (13)娐 Cuon alpinus & 5 & 3 & 54 & 0.03 & EN & 东洋型 \\
\hline \multicolumn{7}{|l|}{ 9. 熊科 Ursidae } \\
\hline (14)马来熊 Helarctos malayanus & 4 & 4 & 14 & 0.02 & EN & 季风型 \\
\hline \multicolumn{7}{|l|}{ 10. 鼣科 Mustelidae } \\
\hline (15)鼣獾 Melogale moschata & 27 & 9 & 60 & 0.16 & LC & 南中国型 \\
\hline (16)黄喉貂 Martes flavigula & 7 & 5 & 22 & 0.04 & LC & 古北型 \\
\hline \multicolumn{7}{|l|}{ IV长鼻目 PROBOSCIDAE } \\
\hline \multicolumn{7}{|l|}{ 11. 象科 Elephantidae } \\
\hline (17)亚洲象 Elephas maximus & 16 & 7 & 458 & 0.09 & EN & 东洋型 \\
\hline \multicolumn{7}{|l|}{ V偶蹄目 APTIODACTYLA } \\
\hline \multicolumn{7}{|l|}{ 12. 猪科 Suidae } \\
\hline (18)野猪 Sus scrofa & 50 & 16 & 350 & 0.29 & LC & 中亚型 \\
\hline \multicolumn{7}{|l|}{ 13. 鹿科 Cervidae } \\
\hline (19)马来水鹿 Cervus equinus & 184 & 23 & 13,364 & 1.07 & LC & 东洋型 \\
\hline (20)赤麇 Muntiacus vaginalis & 179 & 25 & 2,209 & 1.04 & LC & 东洋型 \\
\hline \multicolumn{7}{|l|}{ 14. 牛科 Bovidae } \\
\hline (21)中华嗗羚 Capricornis milneedwardsii & 3 & 1 & 7 & 0.02 & $\mathrm{VU}$ & 东洋型 \\
\hline 15. 鼠鹿科 Tragulidae & & & & & & \\
\hline & 33 & 7 & 113 & 019 & DD & \\
\hline
\end{tabular}

EN：濒危; VU: 易危; NT: 近危; $\mathrm{LC}$ : 无危; DD: 数据缺乏

EN, Endangered; VU, Vulnerable; NT, Near Threatened; LC, Least Concern; DD, Data Deficient 
白德风, 陈颖, 李俊松, 陶庆, 王利繁, 飘优, 时坤. 西双版纳尚勇自然保护区哺乳动物物种多样性. 生物多 样性, 2018, 26 (1): 75-78.

http://www.biodiversity-science.net/CN/10.17520/biods.2017223

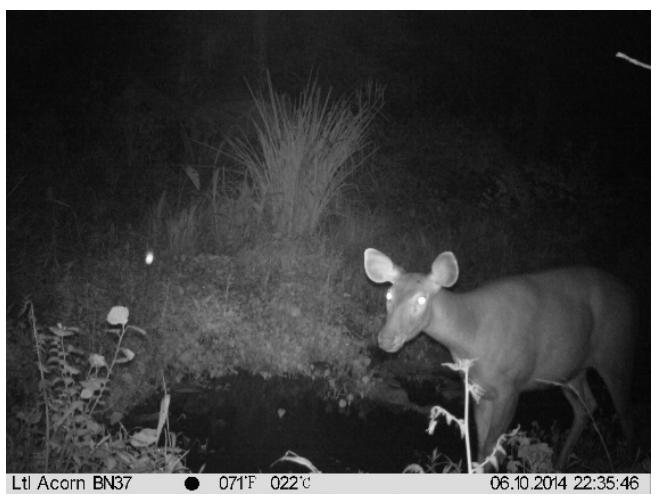

马来水鹿 Cervus equinus

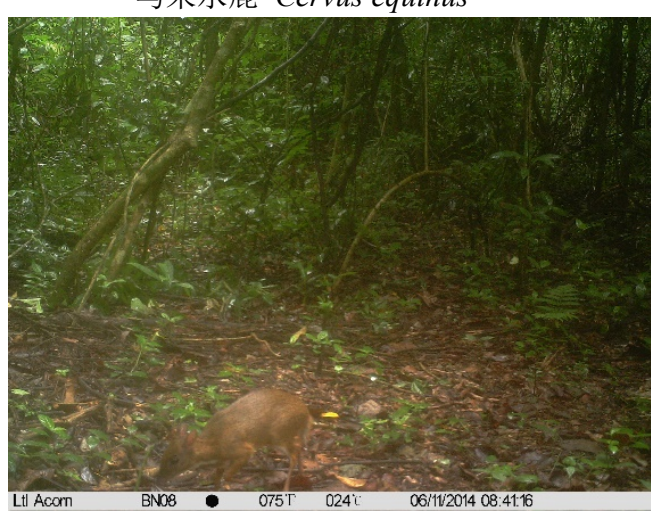

威氏小鼠奚鹿 Tragulus williamsoni

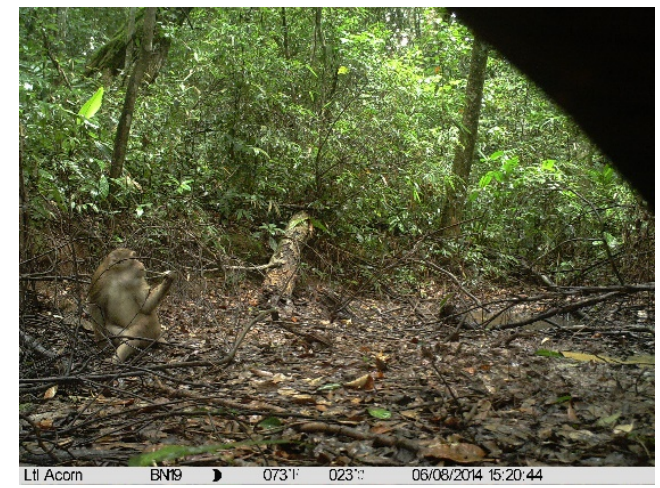

狝猴 Macaca mulatta

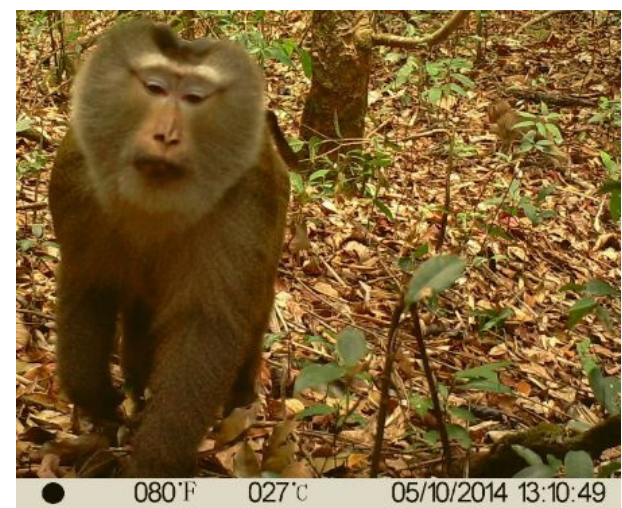

北臀尾猴 Macaca leonina

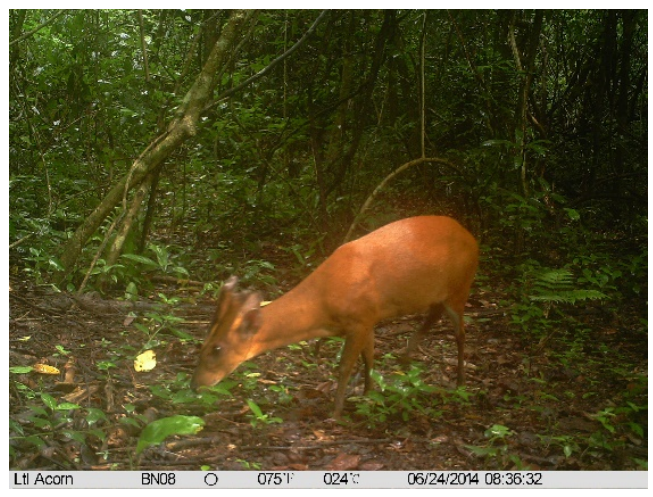

赤鹿 Muntiacus vaginalis

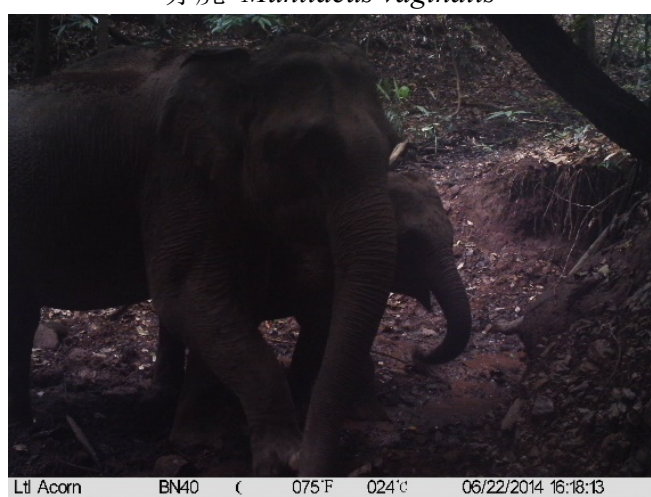

亚洲象 Elephas maximus

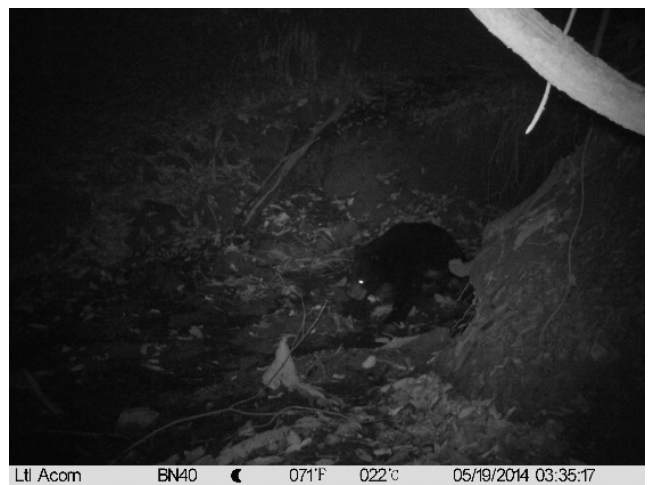

马来熊 Helarctos malayanus

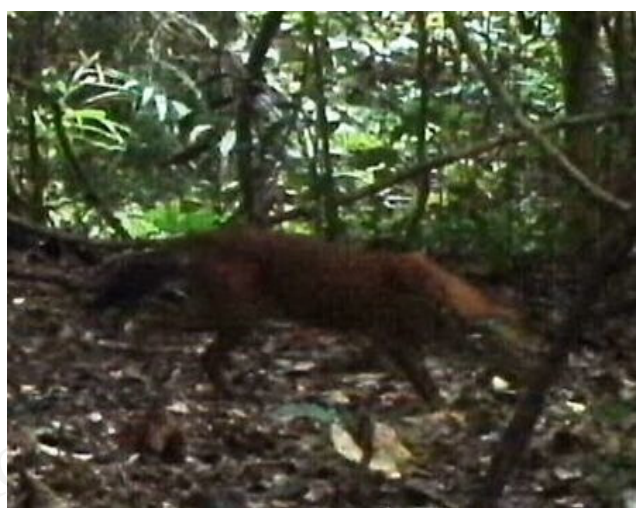

豹 Cuon alpinus

附录2 尚勇自然保护区红外相机拍摄到的重要哺乳动物照片

Appendix 2 Important mammals recorded by camera traps in Shangyong Nature Reserve 